Proceedings

\title{
Crack Resistance of RT-PMMA under Impact Loading ${ }^{\dagger}$
}

\author{
Norazrina Mat Jali 1,2,t, Patrice Longère 1,* \\ 1 Université de Toulouse, ISAE-SUPAERO, ICA (CNRS 5312), 31400 Toulouse, France; \\ Norazrina.BINTI-MAT-JALI@isae.fr \\ 2 Faculty of Engineering, National Defence University of Malaysia, Kuala Lumpur 57000, Malaysia \\ * Correspondence: Patrice.LONGERE@isae.fr; Tel.: +33-561-171-178 \\ † Presented at the 18th International Conference on Experimental Mechanics, Brussels, Belgium, 1-5 July 2018.
}

Published: 19 May 2018

\begin{abstract}
Analysis of failure mechanisms under high strain rate loading in engineering materials is a key point for the design of structures submitted to accidental overloads. We are here interested in the crack arrest capability under impact loading of polymers used as structural and/or protection materials. In the present work, crack arrest capability is defined by how an engineering structure which is initially weakened by a pre-crack behaves when dynamically reloaded. The Kalthoff and Winkler (KW) impact test, consisting in impacting the edge of a double notched plate, is retained for that purpose. An experimental investigation of the dynamic crack arrest capability of shock-resistant PMMA under high strain rate loading is presented, evidencing the brittle feature of the material failure. A high-speed camera is used to record the chronology of the failure mechanisms. It is notably shown that the higher the impact velocity (in the range 50-100 m/s) the larger the number of fragments. Moreover, depending on the impact velocity, changes in the crack path and thus in the mechanisms controlling the PMMA dynamic fracture can be seen.
\end{abstract}

Keywords: impact; fragmentation; RT-PMMA

\section{Introduction}

PMMA is a glassy (amorphous) thermoplastic with excellent optical properties and long term-stability when compared with other tough transparent polymers such as polycarbonate (PC). On the other hand, PMMA has the disadvantage of poor impact (Charpy) toughness, which makes it unsuitable for many engineering applications. Hence, to broaden the range of applications of PMMA, various strategies have been applied. One approach is the addition of well-dispersed rubber particles inside the matrix of PMMA, leading to so called rubber toughened-PMMA or RT-PMMA [1]. With the suitable choice of composition and rubber particles size, this can lead to significant improvements in impact resistance without significantly compromising the optical properties. The role of particle size in particle-filled PMMAs has been extensively discussed in the literature and it is also well recognized that increasing filler content will increase toughness [2-4].

Polymer structural damage can be categorized into macro and microscopic levels. Micro cracking which results from impact and internal stresses is an example of microscopic scale damage. This micro-cracking is the main factor of material failure. Indeed, while being generally undetected, it is considered as inducing structural fragmentations which lead to several properties changes such as loss of strength, stiffness and dimensional stability, see e.g., [5]. Rittel and Maigre [6] also suggested that dynamic crack initiation in PMMA happens due to the nucleation of several micro-cracks along the notch tip. The presence of cracks causes stress/strain concentration and may lead to failure of structure with the cracks interfering in most cases. 
The role of toughening mechanism that occurs in rubber-modified plastics, RT-PMMA in the present case, is to dissipate strain energy that are able to extend an existing flow or crack [7]. This toughening mechanism consists of three stages. The stress applied to the materials leads to the development of micro-crazes around the nanoparticles with resulting elastic and plastic strains on their outer surface. These strains may lead to cavitation around the nanoparticle. The rubbery core which is more ductile deforms and detaches from the rigid shell. This cavitation activates plastic deformation within the area around and between the nanoparticles followed by shear yielding along the crack path. Shear yielding disperses energy which is assumed to favor macroscopic toughening of the material [8].

The present work aims at investigating the crack arrest capability of RT-PMMA under impact loading. In the present work, crack arrest capability is defined by how an engineering structure which is initially weakened by a pre-crack behaves when dynamically reloaded. The Kalthoff and Winkler (KW) impact test consisting in impacting the edge of a double notched plate is accordingly used. An experimental investigation of the dynamic crack arrest capability of shock-resistant RT-PMMA under high strain rate loading is presented, evidencing the brittle feature of the material failure. High speed camera is used to record the chronology of the failure mechanisms.

Section 2 details the experimental method. Results and discussion are reported in Section 3 and conclusions are drawn in Section 4.

\section{Experimental Method}

The material used in this study is highly transparent shock resistance RT-PMMA provided in the form of plate. It consists essentially of polymethyl methacrylate and a tough phase distributed therein which is usually present as a core/shell dispersion. In order to study the thermomechanical characteristics of the RT-PMMA under consideration, tensile tests were carried out using an Instron machine with a maximum capacity of $100 \mathrm{kN}$. The strain is measured using a mechanical extensometer mounted on the specimen.

The objective of this study is to investigate experimentally the crack arrest capability of notched structures made of RT-PMMA under dynamic conditions. Therefore, the edge of double-notched plates are impacted using a method similar to the one proposed by Kalthoff and Wrinkler [9]. All Kalthoff and Wrinkler (KW)-type impact tests were conducted at Institut Clément Ader Lab using gas launchers STIMPACT platform. In order to study the effect of the impact velocity on the crack arrest capability of the PMMA, different impact velocities ranging between 50 and $100 \mathrm{~m} / \mathrm{s}$ are considered. The plate dimensions are $40 \times 82 \times 6 \mathrm{~mm}^{3}$ with notches of $300 \mu \mathrm{m}$-thickness and 20 $\mathrm{mm}$-length. The two initial notches that act as a pre-crack in the structure are machined using a wire saw cutter. Specimens are then placed in a closed chamber (see Figure 1). A 6 m-length gas launcher is used to launch a $20 \mathrm{~mm}$-diameter cylindrical steel projectile. A Photron SA5 high-speed camera is used to observe the projectile/plate interaction at $10^{5} \mathrm{fps}$ (frame per second) and $320 \times 192 \mathrm{pixel}^{2}$ spatial resolution.

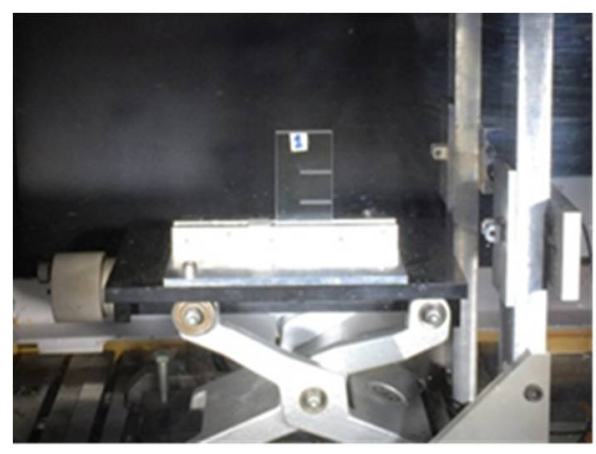

Figure 1. KW-type impact tests. View of the double notched plate in a closed chamber with the support table. The projectile hits the edge of the plate from the right side.

\section{Results and Discussion}


The tension true stress-true strain curves obtained at different strain rates and at room temperature are plotted in Figure 2. As expected, both the Young's modulus and the yield stress are seen to increase with increasing strain rate. The material fails in a ductile manner with a necking process while the strain at failure is increasing with increasing strain rate. This shows the strong dependence of mechanical properties of RT-PMMA on strain rate as in [10]. On the other hand, under tension loading the initially transparent RT-PMMA specimens are subject to progressive stress-whitening, as in $[2,11]$. The stress whitening effect results from rubber particles-PMMA matrix debonding which leads to micro-voiding. The presence of micro-voids provokes light scattering and impairs the initial transparency of the RT-PMMA which progressively becomes opaque.

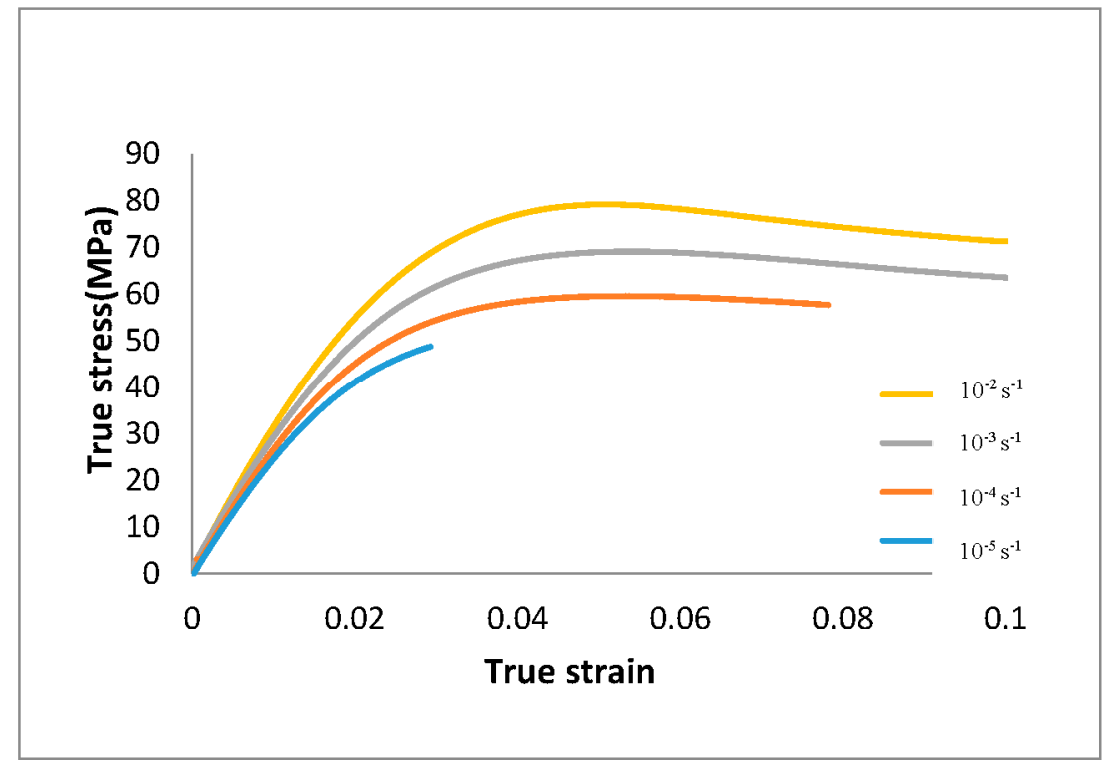

Figure 2. Tension tests for the RT-PMMA under consideration. True stress-true strain curves at room temperature and at different strain rates $\left(10^{-5}\right.$ to $\left.10^{-2} \mathrm{~s}^{-1}\right)$.

Some frames recorded using the high speed camera during the projectile/plate interaction are shown in Figure 3. The frames correspond to different impact velocities and have been recorded at the same time after the impact.

According to Figure 3, whatever the impact velocity, the first cracks initiate from both notches and propagate symmetrically throughout the specimen with an initial angle close to $70^{\circ}$ with respect to the notch direction. This angle is typical of brittle failure controlled by normal stress - in the case of ductile failure the cracks propagate (at least initially) in the notch direction, see [6]. Second cracks initiate from the rear edge of the plates followed by crack branching leading to multi-fragmentation at high velocities.

Stress whitening zones showing areas under tension loading are visible in Figure 3, in particular near the rear side of the specimen (see the frame for $50 \mathrm{~m} / \mathrm{s}$ ) and also at the tip of the first cracks (not shown here). White localization bands are as well visible, initiating from the second cracks (coming from the rear side) and stopping or leading to crack formation in their wake depending on the impact velocity (see frames for $80 \mathrm{~m} / \mathrm{s}$ and $100 \mathrm{~m} / \mathrm{s}$ ).

RT-PMMA plates impacted at $100 \mathrm{~m} / \mathrm{s}$ are damaged giving rise to many fragments of varying sizes. In contrast, for 50 and $80 \mathrm{~m} / \mathrm{s}$, only a few large fragments were produced (see Figure 4). 

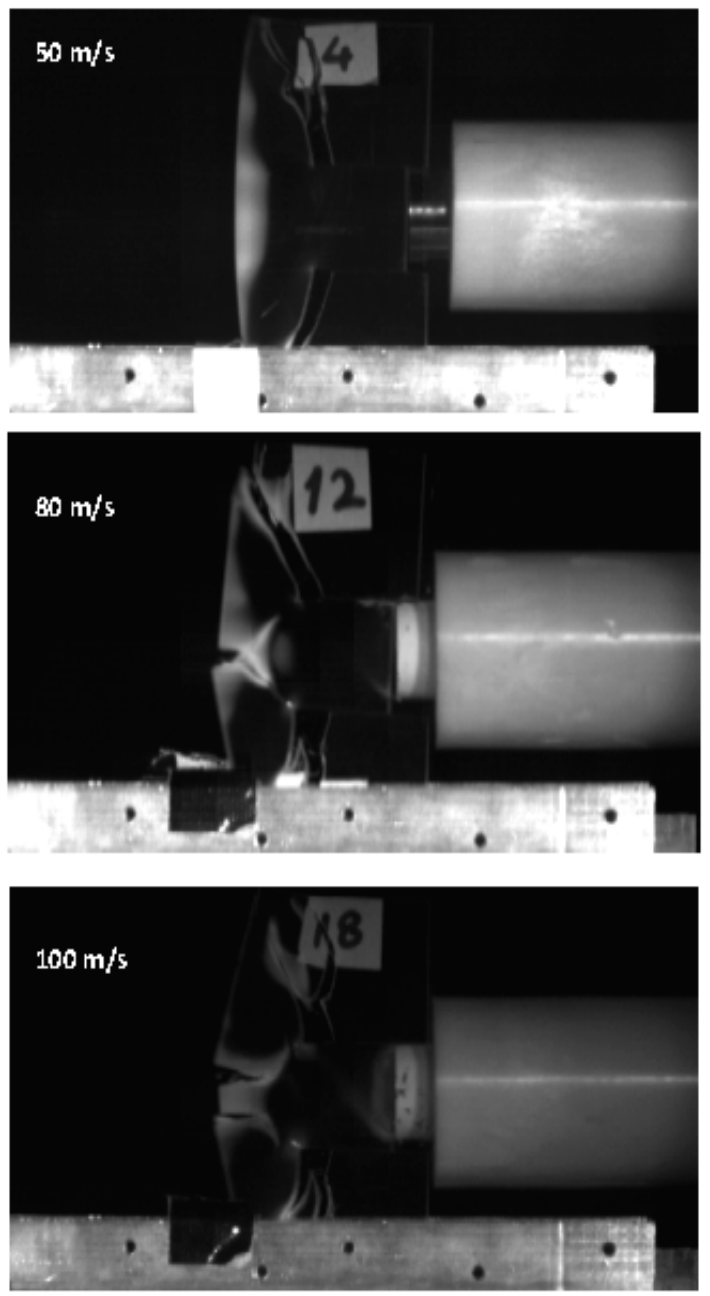

Figure 3. KW-type impact tests for the RT-PMMA under consideration. Three different impact velocities (50 to $100 \mathrm{~m} / \mathrm{s}$ ). Same time after impact.

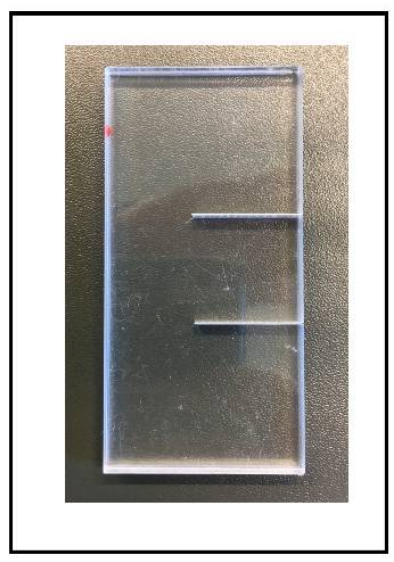

(a)

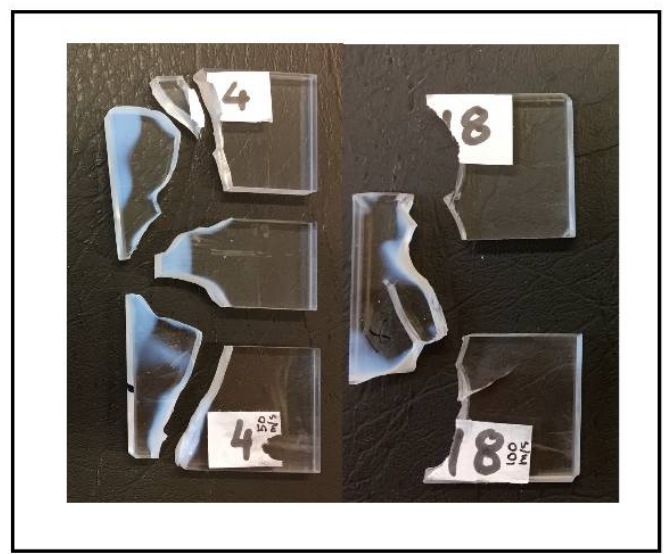

(b)

Figure. 4. KW-type impact tests for the RT-PMMA under consideration. Double notched plates (a) before and (b) after impact loading at $50 \mathrm{~m} / \mathrm{s}$ (left) and $100 \mathrm{~m} / \mathrm{s}$ (right).

The specimens under tension loading and the fragments of impacted plates, see Figure 4, seem to evidence the irreversible feature of the process of micro-voiding induced stress-whitening. However, it can be seen from the frames recorded using the high-speed camera that some regions of the plates may become partially white (or grey) at the passage of the stress waves then recover their transparency after the passage of the stress waves. Thus, micro-voiding induced stress-whitening 
actually seems to be (at least partially) a reversible process. Micro-void opening indeed results from de-bonding which implies the failure of chemical bonds whereas the micro-void closure is merely a gap closure without chemical bonds recovery.

\section{Conclusions}

Crack arrest capability is an important characteristic for a structure especially when the latter undergoes dynamic loading as encountered during accidental events. Addition of rubber to PMMA matrix aids to enhance the toughness by dissipating a part of the impact (kinetic) energy. Low impact velocities result in less number of fragments. The cavitation and internal de-bonding of rubber particles during impact may contribute to dense stress-whitening of some regions of the structure.

Acknowledgments: The authors would like to acknowledge the support of AIRBUS Helicopter.

Conflicts of Interest: The authors declare no conflict of interest.

\section{References}

1. Bagheri, R.; Pearson, R.A. Role of particle cavitation in rubber-toughened epoxies: 1. Microvoid toughening. Polymer 1996, 37, 4529-4538.

2. Lalande, L.; Plummer, C.J.G.; Ge, P.; Ma, J.E. Microdeformation mechanisms in rubber toughened PMMA and PMMA-based copolymers. Polymer 2006, 73, 2413-2426.

3. Plummer,C.J.G.; Béguelin, P.; Kausch, H.H. Microdeformation in core-shell particle modified polymethylmethacrylates. Colloids Surf. A Physicochem. Eng. Asp. 1999, 153, 551-566.

4. Cho, K.; Yang, J.H.; Park, C.E. The effect of rubber particle size on toughening behaviour of rubber-modified poly(methyl methacrylate) with different test methods. Polymer 1998, 39, 3073-3081.

5. Awaja, F.; Zhang, S.; Tripathi, M.; Nikiforov, A.; Pugno, N. Progress in Materials Science Cracks, microcracks and fracture in polymer structures: Formation, detection, autonomic repair. Prog. Mater. Sci. 2016, 83, 536-573.

6. Rittel, D.; Dorogoy, A. Mechanics of Materials Impact of thick PMMA plates by long projectiles at low velocities. Part I: Effect of head's shape. Mech. Mater. 2014, 70, 41-52.

7. Papadopoulos, G.A.; Papanicolaou, G.C. Dynamic crack propagation in rubber-modified composite models. J. Mater. Sci. 1988, 23, 3421-3434.

8. Celestine, A.D.N.; Beiermann, B.A.; May, P.A.; Moore, J.S.; Sottos, N.R.; White, S.R. Fracture-induced activation in mechanophore-linked, rubber toughened PMMA. Polymer 2014, 55, 4164-4171.

9. Roux, E.; Longère, P.; Cherrier, O.; Millot, T.; Capdeville, D.; Petit, J. Analysis of ASB assisted failure in a high strength steel under high loading rate. Mater. Des. 2015, 75, 149-159.

10. Nasraoui, M.; Forquin, P.; Siad, L.; Rusinek, A. Influence of strain rate, temperature and adiabatic heating on the mechanical behaviour of poly-methyl-methacrylate: Experimental and modelling analyses. J. Mater. 2012, 37, 500-509.

11. Jerjen, I.; Revol, V.; Brunner, A.J.; Schuetz, P.; Kottler, C.; Kaufmann, R.; Luethi, T.; Nicoletti, G.; Urban, C.; Sennhauser, U. Detection of stress whitening in plastics with the help of X-ray dark field imaging. Polym. Test. 2013, 32, 1094-1098.

(C) 2018 by the authors. Licensee MDPI, Basel, Switzerland. This article is an open access article distributed under the terms and conditions of the Creative Commons Attribution (CC BY) license (http://creativecommons.org/licenses/by/4.0/). 